\title{
The borderland of migraine with aura: episodic unilateral mydriasis
}

\author{
F. Maggioni $\cdot$ F. Mainardi $\cdot$ M. L. Malvindi • \\ G. Zanchin
}

Received: 7 July 2010/ Accepted: 3 September 2010/Published online: 23 September 2010

(C) Springer-Verlag 2010

\begin{abstract}
We present the case of a patient who had a 3-year history of episodes of transitory unilateral mydriasis with omolateral blurred vision followed by headache. Thereafter, during the last 4 years, the patient developed a migraine with visual aura, without further episodes of transitory mydriasis. We suggest that the transitory mydriasis previously present could be considered as an unusual form of migrainous aura. A possible pathogenetic mechanism is proposed.
\end{abstract}

Keywords Migraine with aura - Unilateral mydriasis . Unusual auras · Anysocoria $\cdot$ Migraine equivalents

\section{Introduction}

Migraine with aura (MA) is a well known and thoroughly studied disease [1]. Auras are characterized by recurrent episodes of transient focal neurologic dysfunction [1]. The clinical presentations of auras, as codified in the ICHD-II 2004 classification [2], include visual aura (by far the most common), followed by sensory and aphasic auras. A motor aura is recognized as a manifestation of a specific type of MA, i.e., hemiplegic migraine [1]. Other transient neurologic phenomena, which are not considered in ICHD-II as auras and are often difficult to classify, have been considered as the "borderland" of migrainous aura [3].

F. Maggioni ( $₫)$ · M. L. Malvindi · G. Zanchin Headache Centre, Department of Neurosciences,

University of Padua, Padua, Italy

e-mail: ferdinando.maggioni@unipd.it

F. Mainardi

Headache Centre, Hospital SS. Giovanni and Paolo,

Venice, Italy
Before developing a MA according to ICHD-II criteria [2], the patient described herein presented with unexplained recurrent episodic of benign unilateral mydriasis (BEUM) with unilateral blurred vision, followed by slight headache, for approximately 3 years.

\section{Case report}

A 24-year-old man presented to our headache center with a 4-year history of MA (ICHD-II criteria) [2]. The patient reported that 7 years before he had suffered for 3 years of transitory episodic blurring of the vision, always in the left eye. In those instances, viewing himself in the mirror, he noticed that the left pupil was larger than the right pupil, with normal reactivity to light. This sign lasted between 5 and $30 \mathrm{~min}$, and was immediately followed by a slight, nonthrobbing, diffuse headache lasting about 3-4 h, without any other symptom or sign. The frequency was regular and occurred about monthly for approximately 3 years. Blurring of the vision developed and resolved gradually in 5-10 min. He was not aware of trigger factors. He underwent a complete blood screening, gadolinium-enhanced magnetic resonance imaging of the brain and neck, extracranial and transcranial Doppler ultrasound, electroencephalography, and transthoracic echocardiography, all of which were normal. The intraocular eye pressure was normal bilaterally, and the visual acuity was 20/20. The ophthalmologic examination including the visual field yielded normal findings as did the physical and neurologic examinations. It was not possible to examine the patient during the attacks since BEUM did not last more than 30 min.

The first visual aura appeared 3 years later, approximately 3-4 months after the last BEUM: this latter never 
occurred after the appearance of MA. He described the auras as small, translucent, gray-colored spots, similar to those which occur after looking at a bright light. They affected the left visual field, developing from the periphery in 5-10 $\mathrm{min}$ and lasting 20-30 $\mathrm{min}$. The headache began almost immediately after resolution of the visual symptoms. The pain was described as severe, alternating unilateral and pulsating. It lasted approximately $6 \mathrm{~h}$, accompanied by photophobia, phonophobia, occasional nausea without vomiting, and was responsive to NSAIDs and triptans. The attacks occurred once in a month. Patient's mother was affected by migraine without aura.

At the follow-up, the patient continues to report his usual, above described, MA attacks, headache being treated successfully with NSAIDs or triptans.

\section{Discussion}

The MA is characterized by recurrent episodes of transient focal neurologic dysfunction; visual aura is the most frequent symptoms, followed by sensory, aphasic, and motor auras [1]. Much less common symptoms include gustatory, olfactory, or auditory (oscillocusis) hallucinations [1] and higher mental function disorders, such as confusional states particularly seen in children [4], temporal lobe phenomena [5], agraphia [6], and alien hand [7].

In clinical practice, other symptoms not particularly rare, and in the past classified as "migraine equivalents" [2], are considered as the borderland of migrainous auras such as recurrent abdominal pain, cyclic vomiting, vertigo, and transient global amnesia [2].These symptoms, because of their modality of presentation, recurrence, length, and the negativity of physical and instrumental examinations, could be considered as "aura phenomena". Uncommon aura presentations are listed in Table 1. In this context, BEUM described in this case could be considered as a possible aura.

In the literature, BEUM has been reported in the past and is described as a primary benign condition [8-11]. Only the paper of Woods et al. [8] focused specifically on BEUM in relation to migraine. They described seven patients with migraine associated with BEUM, four "classic", one "common", one post-traumatic, and one not reported; however, BEUM was not considered as a possible aura, but as a concomitant symptom. Interestingly, also in the other papers dealing with BEUM, this symptom occurs in patients with a personal or familiar history of migraine or headache $[8,9]$. Moreover, there are cases, described in detail and fulfilling ICHD-II criteria, in which we find a transitory recurrent mydriasis, which is considered as an accompanying symptom of migraine without aura [12] or a possible manifestation of ophthalmoplegic migraine (OM)
Table 1 Unusual presentation of aura in migraine

Perceptual symptoms
Visual
Metamorphopsia (distorted shapes)
Achromatopsia (disappearance of colors)
Autokinesis (immobile objects appear in movement)
Macropsia
Micropsia
Paliopsia (visual perseveration)
Prosopoagnosia
Teleopsia
Uditive
Oscilloacusis
Hyperacusis
Gustative hallucinations
Olfactive hallucinations
Neuropsycological complex symptoms
Acalculia
Agraphia
Apraxia
Body schema disorders
Neglect
Acute confusional state
Automatic behavior
Disorders of time perceptions
Déjà vu
Jamais vu
Others
Vertigo
Abdomininal migraine migraine
Carderal episodic mydriasis?

without external involvement of the third cranial nerve $[13,14]$.

The pathogenesis of mydriasis in BEUM could be different from that associated with OM. In fact, mydriasis could be secondary to a hyperactivity of sympathetic or a hypoactivity of parasympathetic system. The occurrence of mydriasis in $\mathrm{OM}$ is considered as secondary to a functional exhaustion of parasympathetic fibers running within the third cranial nerve, with absence of light reflex [14]. OM was considered to have an ischemic cause. More recently, the hypothesis of a demyelinating mechanism affecting the oculomotor nerves has been put forward, following the demonstration by imaging of swelling and enhancement of the intracisternal portion of the third nerve. Upon activation of the trigeminovascular system, neuropeptides secreted at the level circle of Willis could open the bloodnerve barrier causing a sterile inflammation, edema, and 
demyelination of the nerve at its exit from the brainstem [15].

In our case, the recurrence and the length of transitory mydriasis is more suitable with an aura than with an episodic OM. To investigate its pathogenetic mechanism, it would have been interesting to perform a drug test with locally instilled mydriatic/miotic agents in order to distinguish between sympathetic/parasympathetic involvements. Unfortunately, due to the short duration of BEUM, we could not obtain this test from our patient. However, its transitory mydriasis with a preserved light reflex could be related to a central hyperactivity of the sympathetic system starting from the hypothalamic region if we accept the hypothesis that the mechanism underlying the spreading depression could be localized not only in the cerebral cortex but even in other brain areas as suggested by other atypical auras [7].

The differential diagnosis of MA includes transitory ischemic attacks, retinal diseases, partial seizures, and recurrent brain embolism. When headaches present with pupillary dilation, acute third nerve palsies from aneurysms or OM have also to be ruled out [3]. In our patient, a possible secondary cause of mydriasis was carefully evaluated and excluded with neuroimaging and other tests.

In summary, 3 years after presenting recurrent episodes of BEUM followed by non migrainous headache, our patient developed a typical visual aura with a migrainous headache (ICHD-II, 1.2.1). It is difficult to explain this change, but we deem highly improbable that the consecutive occurrence of these two clinical pictures could be simply coincidental: it is not rarely observed that aura could undergo modification during the natural history of the patients without a convincing explanation [7].

A not well-defined transitory blurred vision, often unexplained, is frequently reported by migrainous patients [16], and BEUM can be overlooked and underreported because it is often asymptomatic or accompanied by a slight blurred vision. This kind of clinical manifestation, while very unusual, should be considered when migrainous patients report transitory or not well-defined visual blurred vision not classifiable as typical visual auras: these attacks should be verified as possible BEUM, asking the patients to check for this possibility. Reports on this subject are scarce, and the presentation of this case is intended to stimulate further descriptions, that could suggest to include BEUM in the chapter of MA as an atypical aura.

Conflict of interest None.

\section{References}

1. Olesen J (2006) Migraine with aura. In: Olesen J, Goadsby PJ, Ramadan NM, Tfelt-Hansen P, Welch KM (eds) The headaches, 3rd edn. Lippincott, William \& Wilkins, Philadelphia

2. Headache Classification Subcommittee of the International Headache Society (2004) The international classification of headache disorders, 2nd edn. Cephalalgia 24(S1):1-160

3. Kunkle RS (2005) Migraine aura without headache: benign, but a diagnosis of exclusion. Cleve Clin J Med 72:529-534

4. Gascon G, Barlow C (1970) Juvenile migraine, presenting as an acute confusional state. Pediatrics 45:628-635

5. Peatfield RC (1991) Temporal lobe phenomena during the aura phase of migraine attacks. J Neurol Neurosurg Psychiatry 54:371372

6. Ardilla A, Sanchez E (1988) Neuropsychologic symptoms in migraine syndrome. Cephalalgia 8:67-70

7. Vincent MB, Hadjikhani N (2007) Migraine aura and related phenomena: beyond scotomata and scintillations. Cephalalgia 27:1368-1377

8. Woods D, O'Connor PS, Fieming R (1984) Episodic unilateral mydriasis and migraine. Am J Ophthalmol 98:229-234

9. Jacobson DM (1995) Benign episodic unilateral mydriasis. Clinical characteristics. Ophthalmology 102:1623-1627

10. Edelson RN, Levy DE (1974) Transient benign unilateral pupillary dilation in young adults. Arch Neurol 31:12-14

11. Gilbert ME, Friedman D (2007) Migraine and anisocoria. Surv Ophthalmol 52:209-212

12. Evans RW, Jacobson DM (2003) Transient anisocoria in migraineur. Headache 43:416-418

13. Choi JY, Jang SII, Ho MP, Jo KB, Lee DH (2007) Ophthalmoplegic migraine with alternating unilateral and bilateral internal ophthalmoplegia. Headache 47:726-728

14. Miller NR (1986) Intermittent pupillary dilatation in a young woman. Surv Ophthalmol 31:65-68

15. Bek S, Genc G, Demirkaya S, Eroglu E, Odabasi Z (2009) Ophthalmoplegic migraine. The Neurologist 15:147-149

16. Gupta R, Bhatia MS (2006) A report of cranial autonomic symptoms in migraineurs. Cephalalgia 27:22-28 